\title{
Far-field optical imaging and manipulation of individual spins with nanoscale resolution
}

\author{
P. C. Maurer ${ }^{1 \dagger}$, J. R. Maze ${ }^{1,2 \dagger}$, P. L. Stanwix ${ }^{3,4 \dagger}$, L. Jiang ${ }^{5}$, A. V. Gorshkov ${ }^{1}$, A. A. Zibrov' ${ }^{1}$, B. Harke ${ }^{6}$, \\ J. S. Hodges ${ }^{1,7}$, A. S. Zibrov ${ }^{1}$, A. Yacoby ${ }^{1}$, D. Twitchen ${ }^{8}$, S. W. Hell ${ }^{6}$, R. L. Walsworth ${ }^{1,3}$ \\ and M. D. Lukin ${ }^{1 \star}$
}

A fundamental limit to existing optical techniques for measurement and manipulation of spin degrees of freedom is set by diffraction, which does not allow spins separated by less than about a quarter of a micrometre to be resolved using conventional far-field optics. Here, we report an efficient far-field optical technique that overcomes the limiting role of diffraction, allowing individual electronic spins to be detected, imaged and manipulated coherently with nanoscale resolution. The technique involves selective flipping of the orientation of individual spins, associated with nitrogen-vacancy centres in room-temperature diamond, using a focused beam of light with intensity vanishing at a controllable location, which enables simultaneous single-spin imaging and magnetometry at the nanoscale with considerably less power than conventional techniques. Furthermore, by inhibiting spin transitions away from the laser intensity null, selective coherent rotation of individual spins is realized. This technique can be extended to subnanometre dimensions, thus enabling applications in diverse areas ranging from quantum information science to bioimaging.

O ptical techniques constitute powerful tools for spin detection and manipulation that enable applications ranging from atomic clocks ${ }^{1,2}$ and magnetometers ${ }^{3}$ to quantum information processors ${ }^{4-7}$ and new sensors and imaging modalities for the biological and life sciences ${ }^{8-13}$. Several promising methods for fluorescence imaging have recently been developed to surpass the diffraction limit and are already being applied to important problems in biology and neuroscience ${ }^{14-16}$ as well as subwavelength optical lithography ${ }^{17-19}$. For example, subdiffraction imaging of fluorophores can be obtained by stimulated emission depletion (STED) microscopy and related methods based on reversible saturable optical linear fluorescence transitions ${ }^{20-23}$ (RESOLFT). Using optical fields with intensity zeros and steep spatial gradients, such as those provided by doughnut-shaped beams, one can transiently switch the fluorophores to a different state everywhere except for a small region near the vanishing optical intensity. In this case the emitters from that small region can be separated from neighbours closer than the diffraction limit. As the emitters are switched to the designated (on or off) state provided the optical stimulation rate exceeds that of the spontaneous decay rate of that state, the ultimate resolution is, in principle, limited only by the applicable optical power ${ }^{23}$.

\section{The concept of subdiffraction spin detection and control}

Our new approach to subdiffraction spin detection and manipulation is outlined in Fig. 1. We consider an electronic spin system, such as the nitrogen-vacancy (NV) centre in diamond, which can be polarized by optical pumping, coherently manipulated with resonant microwave radiation and read-out with spin-state-dependent fluorescence. Improved spatial resolution is achieved by illuminating the sample with a doughnut-shaped optical beam just before optical spin readout. Spins positioned directly in the centre of the doughnut beam are not affected by it. However, spins that are even a few tens of nanometres away from the zero-intensity position are repolarized by the doughnut beam and thus contribute differently to the fluorescence signal, providing nanoscale imaging contrast. Moreover, selective coherent manipulation of a spin in the doughnut centre can be achieved by simultaneous exposure to resonant microwave radiation and the optical doughnut beam (Fig. 1c). The essence of this process is that all coherent spin transitions away from the doughnut centre are inhibited by the laser field, thereby allowing coherent rotation of an individual spin at the doughnut centre.

Before proceeding we note that STED and related RESOLFT techniques have been demonstrated to be effective in systems containing metastable states ${ }^{20,21}$, but in all cases electronic excitations were critically employed to resolve at subdiffraction distances. A specific advantage of our present approach, which we refer to as spin-RESOLFT, stems from the exceptionally long lifetimes associated with spin sublevels of electronic ground states in certain systems, which can reach seconds and exceed the lifetimes of electronic transitions by more than six orders of magnitude. Optical transitions involving such ground-state spin levels can be differentially saturated by scattering just a few photons. Therefore, spin-RESOLFT allows individual electronic spins associated with $\mathrm{NV}$ centres to be imaged on the nanoscale, with several orders of magnitude lower laser power than most other super-resolution imaging techniques. This offers significant advantages in many

\footnotetext{
${ }^{1}$ Department of Physics, Harvard University, Cambridge, Massachusetts 02138, USA, ${ }^{2}$ Facultad de Física, Pontificia Universidad Católica de Chile, Casilla 306, Santiago, Chile, ${ }^{3}$ Harvard-Smithsonian Center for Astrophysics, Cambridge, Massachusetts 02138, USA, ${ }^{4}$ School of Physics, University of Western Australia, Crawley, Western Australia 6009, Australia, ${ }^{5}$ Institute for Quantum Information, California Institute of Technology, Pasadena, California 91125, USA, ${ }^{6}$ Max Planck Institute for Biophysical Chemistry, 37077 Gottingen, Germany, ${ }^{7}$ Department of Nuclear Science and Engineering, Massachusetts Institute of Technology, Cambridge, Massachusetts 02139, USA, ${ }^{8}$ Element Six Ltd, Ascot SL5 8BP, UK. ${ }^{\dagger}$ These authors contributed equally to this work. *e-mail: lukin@fas.harvard.edu.
} 
a
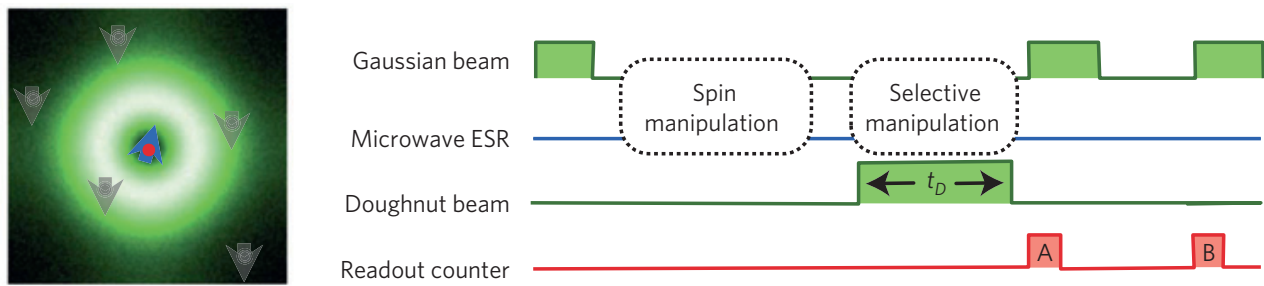

b

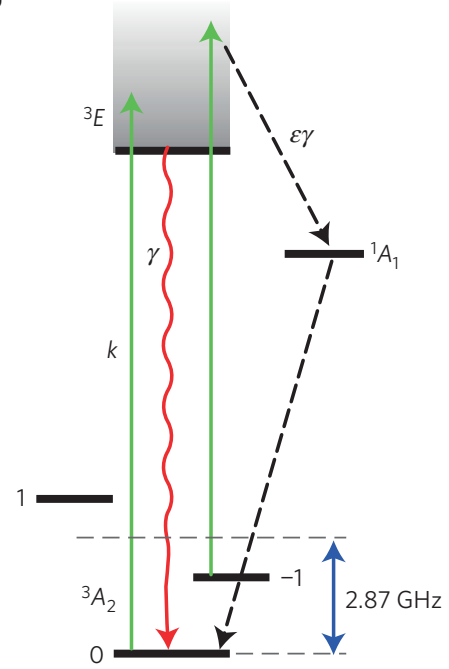

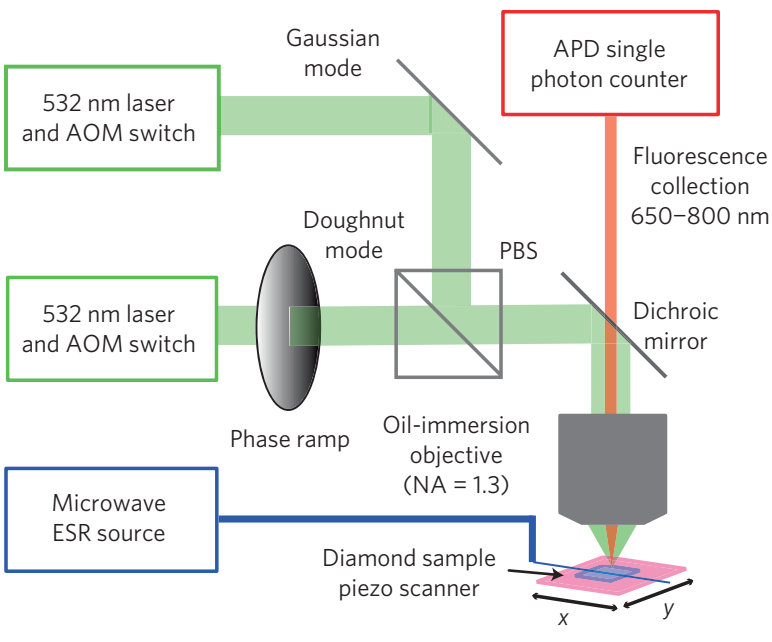

Figure 1 | Principles of subdiffraction far-field optical imaging and manipulation of individual NV electronic spins in diamond (spin-RESOLFT).

a, Experimental sequence using pulsed optical and microwave excitation. NV spins are first optically pumped to $m_{\mathrm{s}}=0$ using a Gaussian beam at $532 \mathrm{~nm}$ followed by microwave manipulation. Application of a $532 \mathrm{~nm}$ doughnut beam repolarizes the outer ring to $m_{\mathrm{s}}=0$, allowing the spin state of the central dark region to be independently read-out. b. Energy-level diagram of an NV centre showing optical absorption $(k)$ and fluorescence $(\gamma)$ rates. NV electronic spins are optically pumped from $\left|m_{\mathrm{s}}\right|=1$ to $m_{\mathrm{s}}=0$ spin states through non-radiative relaxation via the metastable ${ }^{1} A_{1}$ singlet state. Microwave fields at around $2.87 \mathrm{GHz}$ coherently manipulate the NV spin states. The differential spin-state population is measured by spin-state-dependent fluorescence in the red spectral region. c, Schematic of experimental approach. NV centres are imaged using confocal microscopy by scanning the sample around the focal point using a 3 -axis piezo stage. The doughnut beam is generated by passing a Gaussian beam through a vortex waveplate ( $2 \pi$ azimuthally varying phase ramp). APD: avalanche photo detector. See the Methods section and Supplementary Information.

applications, especially to biological systems. Alternatively, for a given power of the doughnut laser beam, spin-RESOLFT allows a marked improvement in resolution relative to STED and other RESOLFT approaches, potentially extending to subnanometre scales. We also note that another method for resolving closely spaced spins employs large magnetic field gradients ${ }^{12}$, as in conventional magnetic resonance imaging. This method, however, requires detailed knowledge of the magnetic gradient topography and is possible only for spins that can be resolved spectrally in electron spin resonance (ESR) or NMR measurements. In what follows we show that the spin-RESOLFT technique allows one to use far-field optical detection to resolve individual spins spaced by subdiffraction distances, sense their local magnetic environment and carry out coherent spin manipulation, even when ESR or NMR measurements are not capable of resolving individual spins spectrally.

\section{Nanoscale optical imaging using spin states}

Our experimental demonstration makes use of individual NV centres in diamond. An electronic spin associated with the NV triplet ground state can be optically polarized, coherently manipulated using conventional ESR techniques ${ }^{24,25}$ and readout through spin-state-dependent fluorescence ${ }^{26}$ (see Fig. 1b). NV centres in ultrapure diamond have exceptional electron spin lifetimes $\left(T_{1}\right)$, approaching seconds at room temperature ${ }^{5,13}$. To realize subdiffraction optical imaging and magnetometry using $\mathrm{NV}$ spins, we first polarize all NV centres in the field of view into the ground spin state $m_{\mathrm{s}}=0$ using a focused Gaussian beam; then drive ESR spin transitions to the $m_{\mathrm{s}}=+1$ or -1 state, or to a coherent superposition of these states; and subsequently use an optical doughnut beam to selectively repolarize the spins of nearby $\mathrm{NV}$ centres. NV centres located in regions of high intensity are optically pumped to the $|0\rangle$ ground state, whereas an NV centre located at the central intensity zero remains unaffected and maintains its original state. Thus, spatial information associated with this central NV is encoded into its electronic spin state. It is subsequently determined by conventional optical readout of the NV fluorescence, because the NV centres that are not repolarized are darker than those that are repolarized. Scanning the sample with respect to the beams and repeating the above procedure allows subdiffraction imaging of the NV centres in the field of view, as well as sensitive nanoscale magnetometry at the location of each NV centre owing to the Larmor precession of a coherent superposition of NV spin states (detectable by spin-state-dependent fluorescence) $)^{9,10}$.

Figure 2 shows example subdiffraction optical images of an NV electronic spin in diamond, acquired using the spin-RESOLFT technique. A tenfold improvement of one-dimensional (1D) resolution was obtained for a fixed power of approximately $2 \mathrm{~mW}$, focused to a diffraction-limited spot of $0.07 \mu \mathrm{m}^{2}$, by increasing the duration of the doughnut beam pulse. A similar resolution improvement was achieved for a much lower doughnut power of about $0.1 \mathrm{~mW}$, provided the decrease in power is compensated by an increase in doughnut duration. These observations are in excellent agreement with predictions from a simple model of the imaging resolution $\Delta r$ 

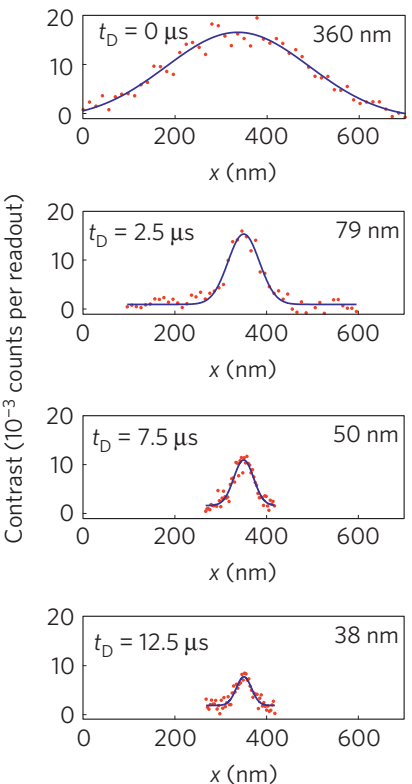

b

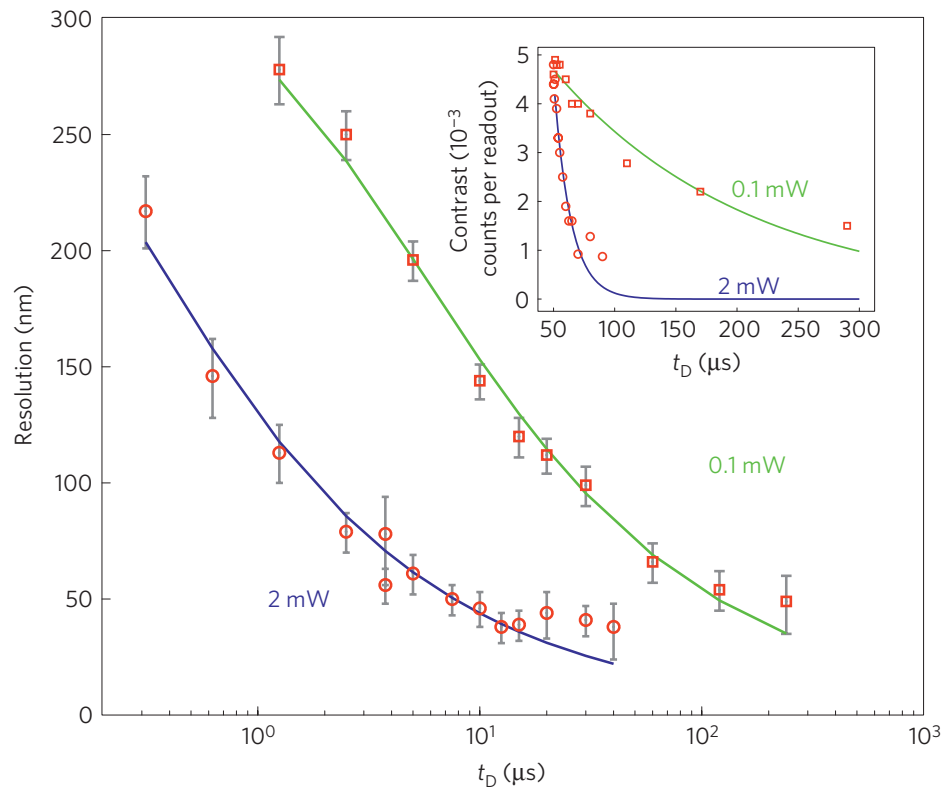

Figure 2 | Demonstration of subdiffraction optical spin imaging. a, Scanning 1D optical images of an NV electronic spin in diamond, for several values of doughnut pulse duration ( $t_{D}$ ) at $2 \mathrm{~mW}$ total doughnut beam power. Contrast is defined as the number of readout counts during the reference readout (period B in Fig. 1) minus the number of counts during the signal (period A). b. Resolution (full-width at half-maximum) of 1D spin images as a function of doughnut pulse duration, for both 2 and $0.1 \mathrm{~mW}$ total doughnut beam power. The error bars represent $95 \%$ confidence intervals. The solid lines represent a fit to equation (1). Equivalent resolution is achieved for lower-power optical fields applied for a longer duration. Long NV spin lifetimes ( $>10 \mathrm{~ms}$ ) enable comparable resolution for the present spin-RESOLFT technique with $\sim 0.1 \%$ of the laser power used in STED imaging of NV centres ${ }^{23}$. Resolution is limited by imperfections of the doughnut intensity zero, and/or mechanical instability during doughnut application, which leads to loss of contrast for very large $t_{D}$ (inset).

provided by our subdiffraction spin detection technique (see the Methods section and Supplementary Information):

$$
\Delta r \approx \frac{\lambda / 2 \mathrm{NA}}{\sqrt{1+\Gamma t_{\mathrm{D}}}}
$$

Here $\lambda$ is the wavelength of the optical field, NA is the numerical aperture of the objective, $\Gamma$ characterizes the maximum rate of optical excitation out of spin state $|1\rangle$ and $t_{\mathrm{D}}$ is the duration of the doughnut beam. Note that the spin-imaging resolution is determined by the product of the power and duration of the doughnut beam: hence subdiffraction-limited resolution can be achieved with a laser power that is well below optical saturation of the excited state $\left({ }^{3} E\right)$, provided that $t_{\mathrm{D}}$ is sufficiently long. As the maximum length of $t_{\mathrm{D}}$ is limited by spin-state relaxation $\left(T_{1}>100 \mathrm{~ms}\right)$ for spin-RESOLFT and by the electronic excited-state lifetime ( $\sim 10 \mathrm{~ns})$ for STED, we are able to realize subdiffraction spin imaging using submilliwatt doughnut beam power with comparable resolution to that of STED using about one watt of doughnut power ${ }^{23}$.

\section{Subdiffraction optical magnetometry}

To demonstrate simultaneous nanoscale spin imaging and magnetometry, we applied the spin-RESOLFT technique to NV centres in bulk diamond separated by less than the diffraction limit. Figure 3a shows subdiffraction 2D imaging of two NV centres with identical ESR lines. In this case, it is not possible to identify the presence of multiple centres using conventional confocal imaging and ESR techniques alone. However, by using our new technique, the presence of two NV centres separated by $\sim 150 \mathrm{~nm}$ is directly revealed in a 2D subdiffraction spin image (Fig. 3b).

Detection of local magnetic fields with subdiffraction resolution is presented in Fig. 3c,d. Specifically, when imaged individually with spin-RESOLFT, each NV centre exhibits a distinct modulation of its Rabi oscillation (Fig. 3c), determined by a relative shift in the centre's ESR transition frequency arising from small differences $(<1 G)$ in the static local magnetic field that each centre experiences. Even more subtle differences in the local magnetic field environment of the two NV centres are revealed by subdiffraction spin-echo measurements using spin-RESOLFT (Fig. 3d). Whereas at short times each of the centres exhibits spin echoes modulated by Larmor precession of the ${ }^{15} \mathrm{~N}$ nuclei in the applied static magnetic field, at longer times the echo signals are substantially different. Centre 2 exhibits good coherent dynamics, evidenced by echo revivals exceeding $20 \mu \mathrm{s}$, whereas centre 1 shows no echo revival, owing to a nearby ${ }^{13} \mathrm{C}$ nucleus ${ }^{27}$ or other magnetic defects (see the Methods section and Supplementary Information). When NV centres have different crystallographic axes and therefore different ESR spectra, the spin-RESOLFT technique can associate each spectral line with the corresponding location of an individual NV centre with subdiffraction-limited resolution (see Supplementary Fig. S1, the Methods section and Supplementary Information).

\section{Coherent spin control}

The spin-RESOLFT technique also enables selective coherent manipulation of individual NV spins separated from other nearby $\mathrm{NV}$ centres by less than the diffraction limit. Applying a strong optical doughnut field simultaneously with a resonant microwave field (Fig. 4a) can suppress coherent spin transitions away from the laser intensity null.

We first demonstrated this effect for an individual, isolated $\mathrm{NV}$ centre, initially polarized into its $m_{\mathrm{s}}=0$ state. To distinguish the optical suppression of microwave-driven spin transitions from the conventional optical process used above for repolarization, we exploited the fact that the optical pumping from $m_{\mathrm{s}}= \pm 1$ into $m_{\mathrm{s}}=0$ occurs via a non-radiative transition through a metastable singlet state on a timescale of about $\tau_{\mathrm{s}}=300 \mathrm{~ns}$. Therefore, we applied a microwave $\pi$-pulse driving the $|0\rangle \rightarrow|1\rangle$ transition together with a simultaneous pulse of the green laser beam; after 
a

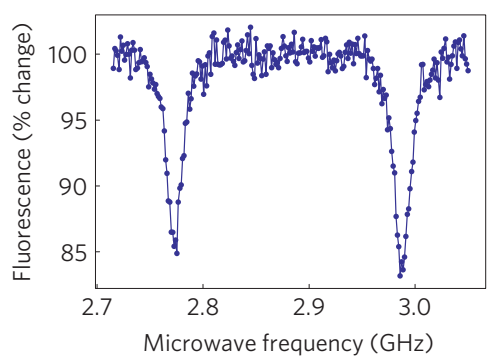

c
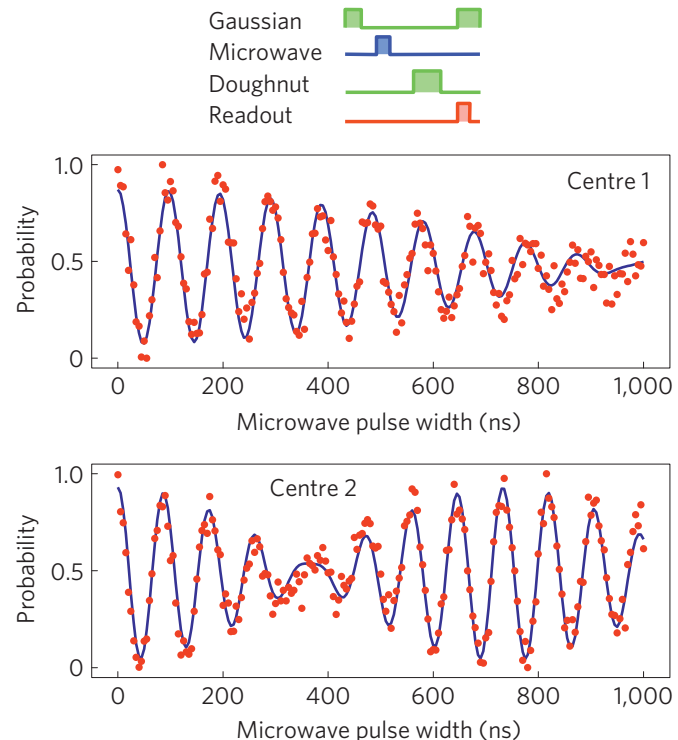
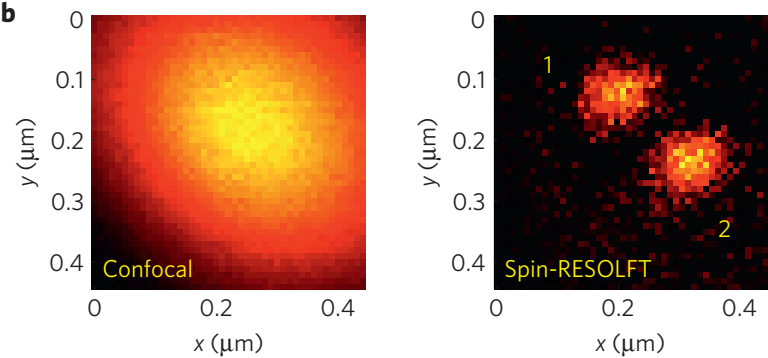

d
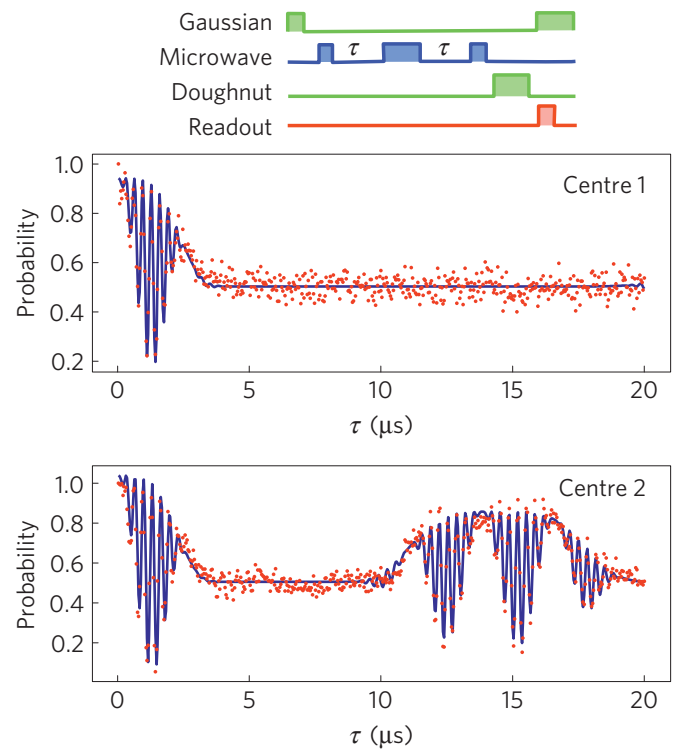

Figure 3 | Subdiffraction optical magnetic sensing. a, Multiple NV centres cannot be identified or distinguished by their optically detected ESR spectrum, acquired using confocal fluorescence microscopy. $\mathbf{b}$, The confocal image does not resolve multiple NV centres, whereas 2D spin imaging using spin-RESOLFT reveals two NV centres separated by $\sim 150 \mathrm{~nm}$. The image has $50 \times 50$ pixels, with a data acquisition time per pixel of $7.2 \mathrm{~s}$. c, Spin-RESOLFT provides spatially selective measurements of Rabi oscillations for the two neighbouring NV spins, which exhibit stark differences owing to different local magnetic field environments. d, Spin-RESOLFT also enables spatially selective spin-echo measurements for the two NV spins, which reveal substantial differences in spin coherence times, indicating the presence of magnetic impurities in the local environment of NV centre 1. The solid lines in $\mathbf{c}$ and $\mathbf{d}$ are fits to a model that includes the electronic spin of the NV centre and the nuclear spin of the centre's ${ }^{15} \mathrm{~N}$ atom.

which we immediately (within $\tau_{\mathrm{d}} \leq 50 \mathrm{~ns} \ll \tau_{\mathrm{s}}$ ) optically measured the populations in the $m_{\mathrm{s}}=0, \pm 1$ and metastable singlet states (see Supplementary Information). As shown in Fig. 4a, as we increased the laser power, the population in each state (triangles) approached the values measured when no microwave pulse was applied (circles). This demonstrates that at high laser power no population transfer occurs between the $|0\rangle$ and $|1\rangle$ states. As our experiment measured the populations before the metastable singlet could decay into the ground state $m_{\mathrm{s}}=0$, the data of Fig. $4 \mathrm{a}$ directly confirm that the observed inhibition of population transfer was not due to repumping out of the $\left|m_{\mathrm{s}}\right|=1$ states.

The observed population dynamics can be due to three effects, all of which result in a suppression of coherent spin manipulation by the applied microwave field. First, if an NV centre is optically promoted to the electronic excited state, then the microwave transition between spin states with $m_{\mathrm{s}}=0$ and $m_{\mathrm{s}}=1$ is suppressed because of the different zero-field splitting in the electronic ground and excited states. Second, if an NV centre is transferred nonradiatively to the metastable ${ }^{1} A_{1}$ state, then coherent spin rotations are prevented because the ${ }^{1} A_{1}$ state is a spin singlet. Finally, optical excitation inhibits the build-up of spin coherence between the $|0\rangle$ and $|1\rangle$ states even in the absence of population transfer to the excited state, a phenomena known as the quantum Zeno effect ${ }^{28}$. To estimate how substantially each of these three processes contributes to the observed suppression of coherent spin transitions, we calculated the populations in the electronic excited state and in the ${ }^{1} A_{1}$ state using the transition rates determined in Fig. 4a. As shown in Supplementary Fig. S4 for our experimental parameters $(R / \gamma \approx 1)$, the estimated population in the ground state is of the same order as the sum of populations in the excited state and the singlet. Thus, population transfer and the quantum Zeno effect contribute approximately equally to the observed suppression of coherent spin manipulation.

We next employed spin-RESOLFT for selective coherent manipulation of one of two closely spaced NV spins (centre 1 in Fig. 3b), while the other NV spin (centre 2) was kept in its $m_{s}=0$ state by optically induced suppression of coherent spin transitions, as discussed above. A pulsed strong doughnut beam was applied simultaneously with a microwave pulse of variable Rabi spin-flip angle, followed by optical measurement of the state of the NV spin at the doughnut centre. In a separate calibration experiment, the states of both NV spins were measured together (no optical doughnut applied) as a function of microwave pulse length (Rabi spin-flip angle). The spin-RESOLFT and calibration measurements enabled us to determine the states of the two proximally spaced NV spins individually. Specifically, two experiments were carried out. In one, the spin states of both NV centres were measured (sequence S3); in the second, the spin state of centre 1 was determined using spin-RESOLFT (sequence S4); see Supplementary Information for details. As shown in Fig. 4b, spin transitions are inhibited for the 
a
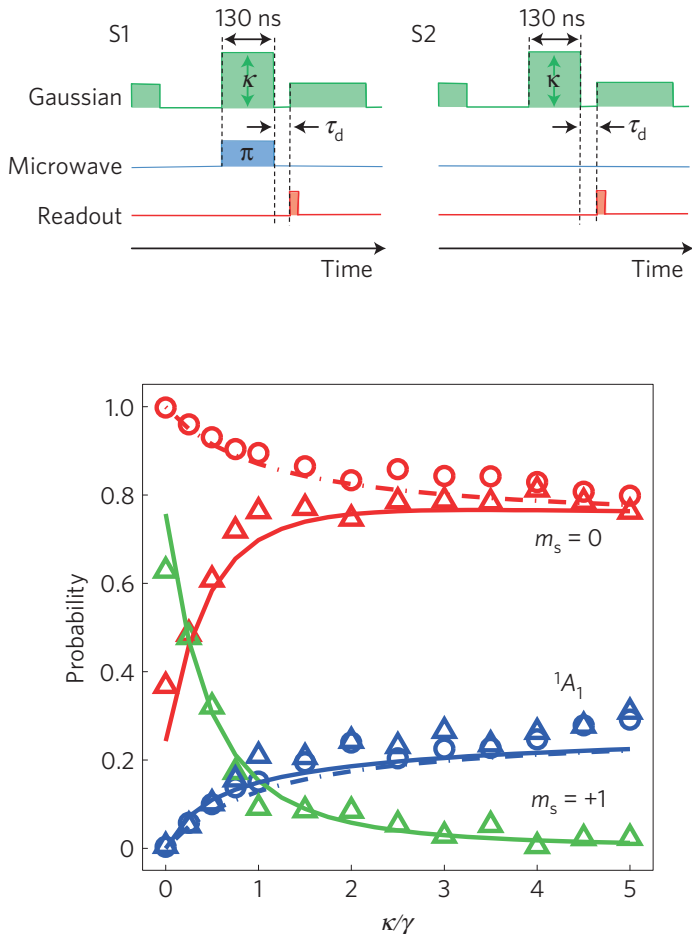
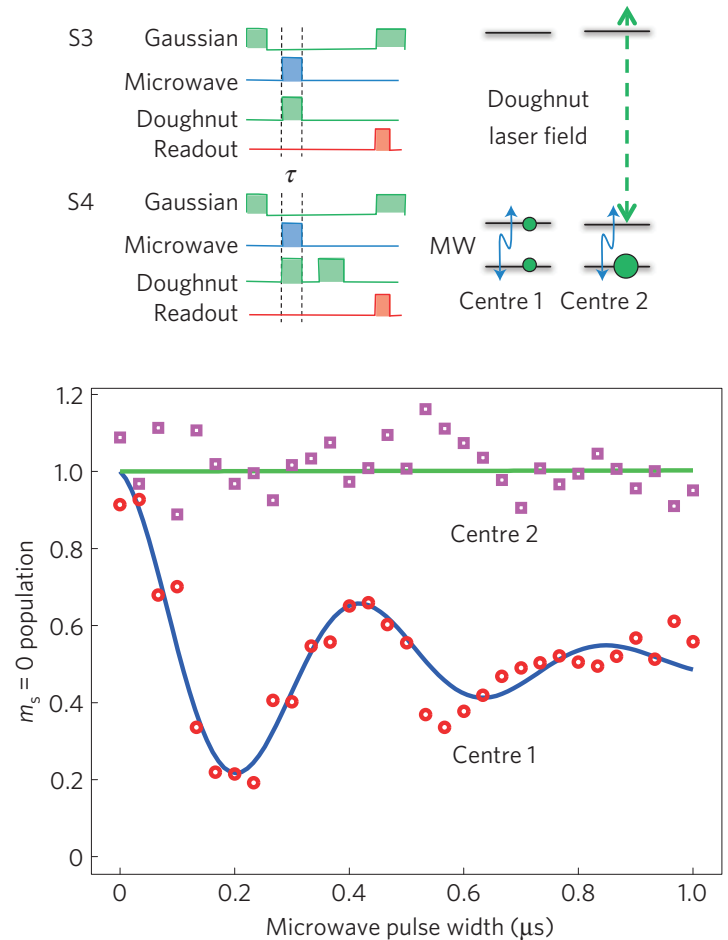

Figure 4 | Subdiffraction coherent manipulation of spectrally indistinguishable NV spins using optically induced effects. a, Suppression of a single, isolated NV centre's spin transitions, driven by a resonant microwave pulse, as a function of Gaussian laser beam power (characterized by the NV optical excitation rate, $\kappa$ ). Using sequences $\mathrm{S} 1$ and $\mathrm{S} 2$, the populations of the $m_{\mathrm{S}}=0$ state (red), $m_{\mathrm{s}}=+1$ state (green) and ${ }^{1} A_{1}$ state (blue) were measured with (triangles) and without (circles) a resonant microwave $\pi$-pulse of 130 ns duration, applied simultaneously with the Gaussian optical beam. The state populations were determined by recording the fluorescence right after microwave pulse application $\left(\tau_{\mathrm{d}}<50 \mathrm{~ns}\right)$ and also after waiting for a time $\left(\tau_{\mathrm{d}}=5 \mu \mathrm{s}\right)$ much longer than the singlet deshelving time ( $\left.300 \mathrm{~ns}\right)$. The comparison between these two measurements allows us to extract the populations of all states, including the singlet (see Supplementary Information). The solid lines are fits using the master equation described in ref. 32 and in the Supplementary Information. b, Coherent spin transitions induced by resonant microwave radiation (MW) are inhibited for NV centres away from the central null of a simultaneously applied green doughnut beam. Selective Rabi oscillations are observed only for the NV spin in the doughnut null (centre 1), whereas NV centre 2, separated from centre 1 by $150 \mathrm{~nm}$, is located outside the doughnut null, and is observed to remain in the ground state. Populations of centres 1 and 2 are obtained from experimental sequences S3 and S4 as described in the Supplementary Information.

NV centre outside the central null of the doughnut beam, whereas the central NV spin undergoes coherent evolution, that is, Rabi oscillations, in excellent agreement with a theoretical model (blue and green curves in Fig. 4b), as described in the Methods section and Supplementary Information.

\section{Outlook}

As shown in these demonstration experiments, the far-field optical resolution for spin imaging and coherent manipulation is no longer limited by diffraction. We expect the resolution and speed of the spin-RESOLFT technique to be significantly improved by technical upgrades such as increasing the contrast between the intensity of the doughnut crest and that of the central minimum, that is, perfecting the doughnut zero ${ }^{23}$. In addition, implementing relatively large values of $\Gamma t_{\mathrm{D}}$, where $\Gamma \sim 100 \mathrm{MHz}$ and $t_{\mathrm{D}}$ is of the order of the electronic spin lifetime $T_{1}$ (which ranges from $10 \mathrm{~ms}$ (ref. 5) to longer than a second in ultrapure diamond ${ }^{13}$ ), should allow more than a 1,000-fold improvement of resolution below the diffraction barrier, making it feasible to attain subnanometre optical spin detection and manipulation, including sensitive measurement of magnetic fields. Finally, the low levels of light required for our technique will also facilitate the parallelization of this imaging process by replacing the scanning doughnut beam with moving arrays of lines or doughnuts of vanishing intensity. This modification should enable fast camera-based spin imaging over a large field of view.

The capability to optically detect and manipulate individual spins with subdiffraction resolution opens the door to a number of powerful applications in both the physical and life sciences. For example, spin-RESOLFT could be used for high-fidelity addressing of individual spin qubits in an array composed from $\mathrm{NV}$ centres separated by distances in the range of $10-30 \mathrm{~nm}$. In such a case, near electronic spins could be coupled directly through magnetic or optical dipole-dipole interactions, thereby enabling high-fidelity two-qubit operations. By encoding qubits into nuclear spins near to specific NV centres, and using spinRESOLFT for subdiffraction addressing of electronic spins and control of electron-nuclear systems, both readout and coherent manipulation could be enabled on nanometre scales, thereby opening a new route to a room-temperature scalable quantum information processor (see also ref. 29). More specifically, the demonstrated suppression of electronic Rabi oscillations (Fig. 4b) allows for preparation, detection and coherent manipulation of one nuclear spin (associated with the NV centre in the zero of the green doughnut-shaped beam) without affecting qubits encoded in the nuclear spins of surrounding centres ${ }^{30,31}$ (see the Methods section and Supplementary Information). Likewise, intriguing applications in bioscience can be foreseen for spinRESOLFT, which combines high-sensitivity magnetometry with subdiffraction imaging resolution. Examples include the use of $\mathrm{NV}$-diamond nanocrystals in living cells as photostable fluorescent biomarkers and sensors of nanoscale magnetic fields, for example, for direct imaging of activity in neuronal networks and mapping the local concentration of reactive oxygen species and intra-cellular ions. In addition, by exploiting long-lived ground electronic spin 
states, spin-RESOLFT has the potential for reaching extraordinary imaging and sensing resolution with modest laser power levels, which may be of interest for a diversity of super-resolution imaging applications involving, in particular, biological systems. Finally, we expect that our approach can be applicable to a wide variety of spin systems, from trapped atoms and ions to quantum dots, which may enable a diverse range of interesting applications in quantum science and engineering.

\section{Methods}

Samples. All described experiments were carried out at room temperature on an ultrapure single-crystal bulk diamond grown by chemical vapour deposition. $\mathrm{NV}$ centres were created by irradiating the chemical-vapour-deposited diamond with $10^{9} \mathrm{~cm}^{-2}$ of nitrogen ions with an energy of $6 \mathrm{keV}$, corresponding to an estimated average implantation depth of $10 \mathrm{~nm}$, as determined by simulations. The sample was then annealed at $800^{\circ} \mathrm{C}$, yielding an observed $\mathrm{NV}$ concentration of approximately $0.6 \mathrm{NV} \mu \mathrm{m}^{-2}$. To reliably distinguish shallow implanted ions from natural $\mathrm{NV}$ centres, a rare isotope ${ }^{15} \mathrm{~N}$ was used.

Confocal and spin-RESOLFT microscopy. NV centres were probed using a custom-built confocal fluorescence microscope. Two $532 \mathrm{~nm}$ laser beams provided optical excitation, one a Gaussian-shaped beam and the other a doughnut-shaped beam. The two beams, which could be independently switched using acousto-optic modulators (AOMs; Crystal Technology and Isomet), were combined on a polarized beam splitter before entering an oil-immersion objective (Nikon Plan Fluor $100 \times 1.3 \mathrm{NA}$ ). Fluorescence from the NV centres was collected back through the objective, separated from the excitation path by a custom dichroic with high surface flatness (Chroma technology). The fluorescence was filtered (630-750) $\mathrm{nm}$ and focused onto a single-mode fibre connected to an avalanche photo detector (Perkin Elmer). Imaging was achieved by scanning the diamond sample under the objective, using a digitized 3-axis piezo translation stage (Physical Instrument P-733.3CD). The doughnut-shaped mode was created by passing a Gaussian beam through the centre of a helical phase ramp (RPC Photonics), which adds a phase from $(0$ to $2 \pi)$ conditional on the azimuthal position. To prevent distortion of the doughnut centre owing to beam focusing by the objective, the beam was circularly polarized by placing a quarter-lambda waveplate immediately before the objective.

Electronic spin manipulation. The electronic ground state of the NV centre is a spin triplet state, with a zero-field splitting of $2.87 \mathrm{GHz}$ between $m_{\mathrm{s}}=0$ and $m_{\mathrm{s}}= \pm 1$ owing to the electron-electron interaction. Optical excitation with green light optically pumps population from the $m_{\mathrm{s}}= \pm 1$ state into the $m_{\mathrm{s}}=0$ state at a rate $R$. The degeneracy of the $m_{\mathrm{s}}= \pm 1$ state was lifted by Zeeman splitting, induced by an external d.c. magnetic field as well as local magnetic fields to be detected by magnetometry pulse sequences. Moreover, by adjusting the direction of the external magnetic field, the relative ESR frequency for NV centres with different crystallographic orientations was controlled. The NV electronic spin state was coherently manipulated (for Rabi oscillations, spin-echo measurements and so on) by driving the electron spin resonance with a microwave field generated from a $20-\mu \mathrm{m}$-diameter wire placed on the surface of the diamond sample.

Spin-imaging resolution. If a microwave $\pi$-pulse is applied between doughnut and readout the spin-RESOLFT signal is proportional to $f(x)=n_{0}(x) I_{\mathrm{G}(x)}$, where $n_{0}$ is the population of the $m_{\mathrm{s}}=0$ state, $I_{\mathrm{G}}=\exp \left(-\alpha\left(x / r_{\mathrm{C}}\right)^{2}\right)$ is the intensity profile of the Gaussian beam used for readout of the electronic spin, $r_{\mathrm{C}}=\lambda / 2 \mathrm{NA}$ is the confocal resolution and $\alpha=4 / \log (2) . n_{0}$ depends on the duration of the doughnut beam $t_{\mathrm{D}}$; it can be evaluated by solving rate equations given in the Supplementary Information. In the limit of large lifetime of spin transition, it is given by $n_{0}=\exp \left(-R_{x} t_{\mathrm{D}}\right)$, where $R_{x}$ is the position-dependent optical polarization rate. In the unsaturated regime, $R_{x}=R_{0}+\alpha \Gamma\left(x / r_{\mathrm{C}}\right)^{2}$, where $\Gamma=\kappa \epsilon$ is proportional to the optical excitation rate associated with the maximal intensity of the doughnut $\kappa$, and $\epsilon$ is the branching ratio of the spin-changing $\left(m_{\mathrm{s}}=1 \rightarrow m_{\mathrm{s}}=0\right)$ decay relative to the total decay of the excited state. $R_{0}=\epsilon \kappa_{0}$ is proportional to the intensity at the doughnut centre and the corresponding excitation $\kappa_{0}$. The spin-RESOLFT resolution $r$ is defined by $f(x)=\exp \left(-\alpha(x / r)^{2}\right)$. Solving for $r$ leads to equation (1). In our experiments, the resolution is limited by small imperfection of the doughnut zero intensity. As shown in the Supplementary Information, using a model that includes a back pumping process that brings the electronic spin from the $m_{\mathrm{s}}=0$ to the $m_{\mathrm{s}}=1$ state and finite spin lifetime, we find that the improvement in resolution relative to the diffraction limit of a confocal microscope is given by $r / r_{\mathrm{C}} \sim \sqrt{R_{0} / \Gamma} \sqrt{1+2 \gamma_{0 \rightarrow 1} / R_{0}} \approx \sqrt{R_{0} / \Gamma}$, where $\gamma_{0 \rightarrow 1}$ is the total rate out of the electronic spin state $m_{\mathrm{s}}=0$. This estimate is in good agreement with experimental observations.

Measurements of local magnetic field environment. The observed modulations of the Rabi oscillations shown in Fig. $3 \mathrm{c}$ reveal the different magnetic field experienced by each NV centre. These modulations are caused by hyperfine-induced splitting in the NV electronic spin transition $\left(m_{\mathrm{s}}=0 \rightarrow 1\right)$ associated with the ${ }^{15} \mathrm{~N}$ nuclear spin
$(I=1 / 2)$. Such modulations appear when the microwave frequency is detuned from the central transition $v_{0}^{i}=\Delta+\gamma_{\mathrm{e}} B^{i}$, where $B^{i}$ is the magnetic field along the $\mathrm{NV}$ axis of centre $i, \Delta$ is the zero-field splitting and $\gamma_{\mathrm{e}}$ is the gyromagnetic ratio of the electronic spin. The modulation frequency for NV centre $i$ is thus given by (see Supplementary Information) $\left(v_{0}^{i}-v\right) A / \Omega$, where $v$ is the microwave frequency, $A=3.05 \mathrm{MHz}$ is the hyperfine interaction between the electronic and nuclear spin and $\Omega$ is the Rabi frequency. The difference in the beating frequencies of each centre reveals a difference in effective static magnetic field experienced by each centre of about $1 \mathrm{G}$.

In our spin-echo measurements (Fig. 3d), the dominant contribution comes from the interaction between the electronic spin of the NV centre and the ${ }^{15} \mathrm{~N}$ nuclear spin and ${ }^{13} \mathrm{C}$ nuclear spin bath. The high-frequency oscillations $(3.05 \mathrm{MHz})$ correspond to the hyperfine interaction $A$ between the electronic spin and nuclear spin of the ${ }^{15} \mathrm{~N}$ atom in the NV centre, as discussed above. Meanwhile the slow-frequency component of the dynamics $(360 \mathrm{kHz})$ corresponds to the Larmor frequency of the ${ }^{15} \mathrm{~N}$ nuclear spin in the local magnetic field, which is enhanced by virtual transitions of the electronic and nuclear spin (Supplementary Information). The enhancement factor can be as large as $2\left(\gamma_{e} / \gamma_{n}\right)(A / \Delta) \approx 14$ when the magnetic field is perpendicular to the NV axis. The observed spin echo collapses and revivals are due to the interaction between $\mathrm{NV}$ centres and ${ }^{13} \mathrm{C}$ nuclei. The revival frequency in Fig. $3 \mathrm{~d}, 74 \mathrm{kHz}$, is given by the bare Larmor frequency of ${ }^{13} \mathrm{C}$ corresponding to a magnetic field of $69 \mathrm{G}$ (Supplementary Information). Note that centre 1 shows no revival, which is probably due to a proximal ${ }^{13} \mathrm{C}$ nuclear spin that quickly decoheres the electronic spin of this centre ${ }^{23}$.

Coherent single-spin manipulation. We carried out selective coherent spin manipulation on two NV centres with a spatial separation of approximately $150 \mathrm{~nm}$. To verify that centre 1 can undergo coherent Rabi oscillations while centre 2 is kept in the $m_{\mathrm{s}}=0$ state, two experiments using pulse sequences S3 and S4 in Fig. 4 were carried out. If centre 2 remains in the $m_{\mathrm{s}}=0$ state, a constant signal is expected for the difference of the measurements made with these sequences.

In this subtraction procedure we accounted for contrast reduction in the spin-RESOLFT readout of centre 1 by multiplying the results of sequence $S 4$ by $1 / 0.75$. (This weighting factor is determined from a separate experiment, in which both centres were prepared in the state $m_{\mathrm{s}}=1$ and imaged with spin-RESOLFT.) The signals were normalized relative to the unperturbed Rabi oscillation signal for centre 1.

The observed subdiffraction suppression of coherent spin transitions can be used to manipulate quantum information stored in the nuclear spins associated with each NV centre. Specifically, we can use this subdiffraction inhibition to prepare, coherently manipulate and detect the nuclear spin of centre 1 (in the doughnut centre) without perturbing the nuclear spins of centre 2 and of other nearby centres. As explained in detail in the Supplementary Information, the error induced on the nuclear spin associated with centre 2 during a $\pi$-pulse on centre 1 can be estimated from our measurements as $P_{\mathrm{e}} \approx(\gamma /(\kappa+\gamma))(\pi \Omega / 2 \kappa)$, where $\kappa$ is evaluated at centre 2 and where $\gamma \approx 1 / 13$ ns is the decay rate of the optically excited state. In addition to the dephasing described above, this takes into account the fact that application of the green doughnut beam 'hides' some population in the excited state. For values of $\kappa \sim \gamma$ and $\Omega=(2 \pi) 2.3 \mathrm{MHz}$, this error would be $p_{\mathrm{e}} \approx 0.1 \ll 1$, indicating that the state of the nuclear spin associated with centre 2 can be well preserved while we manipulate centre 1 .

Received 5 November 2009; accepted 9 August 2010; published online 19 September 2010

\section{References}

1. Jacques Vanier, C. A. The Quantum Physics of Atomic Frequency Standards (Hilger, 1989).

2. Cundiff, S. T. \& Ye, J. Colloquium: Femtosecond optical frequency combs. Rev. Mod. Phys. 75, 325-342 (2003).

3. Budker, D. \& Romalis, M. Optical magnetometry. Nature Phys. 3, 227-234 (2007)

4. Xu, X. D. et al. Coherent optical spectroscopy of a strongly driven quantum dot. Science 317, 929-932 (2007).

5. Dutt, M. V. G. et al. Quantum register based on individual electronic and nuclear spin qubits in diamond. Science 316, 1312-1316 (2007).

6. Hennessy, K. et al. Quantum nature of a strongly coupled single quantum dot-cavity system. Nature 445, 896-899 (2007).

7. Neumann, P. et al. Multipartite entanglement among single spins in diamond. Science 320, 1326-1329 (2008).

8. Chernobrod, B. M. \& Berman, G. P. Spin microscope based on optically detected magnetic resonance. J. Appl. Phys. 97, 014903 (2005).

9. Taylor, J. M. et al. High-sensitivity diamond magnetometer with nanoscale resolution. Nature Phys. 4, 810-816 (2008).

10. Maze, J. R. et al. Nanoscale magnetic sensing with an individual electronic spin in diamond. Nature 455, 644-647 (2008).

11. Degen, C. L. Scanning magnetic field microscope with a diamond single-spin sensor. Appl. Phys. Lett. 92, 243111 (2008). 
12. Balasubramanian, G. et al. Nanoscale imaging magnetometry with diamond spins under ambient conditions. Nature 455, 648-651 (2008).

13. Balasubramanian, G. et al. Ultralong spin coherence time in isotopically engineered diamond. Nature Mater. 8, 383-387 (2009).

14. Westphal, V. \& Hell, S. W. Nanoscale resolution in the focal plane of an optical microscope. Phys. Rev. Lett. 94, 143903 (2005).

15. Zhuang, X. Nano-imaging with STORM. Nature Photon. 3, 365-367 (2009).

16. Betzig, E. et al. Imaging intracellular fluorescent proteins at nanometer resolution. Science 313, 1642-1645 (2006).

17. Li, L. J., Gattass, R. R., Gershgoren, E., Hwang, H. \& Fourkas, J. T. Achieving $\lambda / 20$ resolution by one-color initiation and deactivation of polymerization. Science 324, 910-913 (2009).

18. Scott, T. F., Kowalski, B. A., Sullivan, A. C., Bowman, C. N. \& McLeod, R. R. Two-color single-photon photoinitiation and photoinhibition for subdiffraction photolithography. Science 324, 913-917 (2009).

19. Johnson, K. S. et al. Localization of metastable atom beams with optical standing waves: Nanolithography at the Heisenberg limit. Science 280, 1583-1586 (1998).

20. Hell, S. W. \& Wichmann, J. Breaking the diffraction resolution limit by stimulated emission: Stimulated-emission-depletion fluorescence microscopy. Opt. Lett. 19, 780-782 (1994).

21. Hell, S. W. Far-field optical nanoscopy. Science 316, 1153-1158 (2007).

22. Hell, S. W \& Kroug, M. Ground-state-depletion fluorescence microscopy: A concept for breaking the diffraction resolution limit. Appl. Phys. B 60, 495-497 (1995).

23. Rittweger, E., Han, K. Y., Irvine, S. E., Eggeling, C. \& Hell, S. W. STED microscopy reveals crystal colour centres with nanometric resolution. Nature Photon. 3, 144-147 (2009).

24. Hanson, R. \& Awschalom, D. D. Coherent manipulation of single spins in semiconductors. Nature 453, 1043-1049 (2008)

25. Hanson, R., Dobrovitski, V. V., Feiguin, A. E., Gywat, O. \& Awschalom, D. D. Coherent dynamics of a single spin interacting with an adjustable spin bath. Science 320, 352-355 (2008).
26. Wrachtrup, J. \& Jelezko, F. Processing quantum information in diamond. J. Phys. Condens. Matter 18, S807-S824 (2006).

27. Maze, J. R., Taylor, J. M. \& Lukin, M. D. Electron spin decoherence of single nitrogen-vacancy defects in diamond. Phys. Rev. B 78, 094303 (2008).

28. Itano, W. M., Heinzen, D. J., Bollinger, J. J. \& Wineland, D. J. Quantum zeno effect. Phys. Rev. A 41, 2295-2300 (1990).

29. Gorshkov, A. V., Jiang, L., Greiner, M., Zoller, P. \& Lukin, M. D. Coherent quantum optical control with subwavelength resolution. Phys. Rev. Lett. 100, 093005 (2008).

30. Neumann, P. et al. Single-shot readout of a single nuclear spin. Science 329, 542-544 (2010).

31. Jiang, L. et al. Coherence of an optically illuminated single nuclear spin qubit. Phys. Rev. Lett. 100, 073001 (2008).

32. Manson, N. B., Harrison, J. P. \& Sellars, M. J. Nitrogen-vacancy centre in diamond: Model of the electronic structure and associated dynamics. Phys. Rev. B 74, 104303 (2006).

\section{Acknowledgements}

We gratefully acknowledge P. Cappellaro, Y. Chu, S. Folling, M. Greiner, P. Hemmer, E. Rittweger, B. Shields, E. Togan, A. Trifonov and D. Wildanger for valuable discussions and technical assistance. This work was supported by the NSF, DARPA, the Packard Foundation, the Smithsonian Institution and Harvard CNS.

\section{Author contributions}

All authors contributed to all aspects of this work.

\section{Additional information}

The authors declare no competing financial interests. Supplementary information accompanies this paper on www.nature.com/naturephysics. Reprints and permissions information is available online at http://npg.nature.com/reprintsandpermissions. Correspondence and requests for materials should be addressed to M.D.L. 